\title{
Improvement of accuracy and speed of a commercial AFM using Positive Position Feedback control
}

\author{
I. A. Mahmood and S. O. R. Moheimani
}

\begin{abstract}
The Atomic Force Microscope (AFM) is a device capable of generating topographic images of sample surfaces with extremely high resolutions down to the atomic level. It is also being used in applications that involve manipulation of matter at a nanoscale. Early AFMs were operated in open loop. As a result, they were susceptible to piezoelectric creep, thermal drift, hysteresis nonlinearity and scan-induced vibration. These effects tend to distort the generated image. The distortions are often minimized by limiting the scanning speed and range of the AFMs. Recently a new generation of AFMs has emerged that utilizes position sensors to measure displacements of the scanner in three dimensions. These AFMs are equipped with feedback loops that work to minimize the adverse effects of hysteresis, piezoelectric creep and thermal drift on the obtained image using standard PI controllers. These feedback controllers are often not designed to deal with the highly resonant nature of an AFM's scanner, nor with the crosscoupling between various axes. In this paper we illustrate the drastic improvement in accuracy and imaging speed that can be obtained by proper design of a feedback controller. Such controllers can be incorporated into most modern AFMs with minimal effort since they can be implemented in software with the existing hardware.
\end{abstract}

\section{INTRODUCTION}

The Atomic Force Microscope, AFM [1], [2] was conceived to generate 3D images of material surfaces with very high accuracies. As illustrated in Fig. 1 in this device, a micro-cantilever, with a very sharp tip, is scanned over a sample at distances of the order of a few nanometers, or less. Interatomic forces between the tip and the sample force the micro-cantilever to deflect. This deflection is measured by a laser and a photodetector. The force experienced by the micro-cantilever is a nonlinear function of the tip-sample separation [2]. This enables the AFM to operate in a number of modes. In contact mode, illustrated in Fig. 1, the tip experiences a repulsive force, which is kept constant during the scan by a feedback loop. The controller is often a PI compensator. In this mode of operation the tip exerts a relatively large normal force, and a considerable lateral force on the sample. Consequently, the probe is subject to significant wear. Hence, this mode may not be suitable for soft samples that can be damaged easily, e.g. biological samples.

A critical component of an AFM is its scanning head that moves either the sample, or the probe in a raster pattern in the $\mathrm{x}$-y plane. It also controls the distance between the probe and the sample through the z-axis servo. The use of a

I. A. Mahmood and S. O. R. Moheimani are with School of Electrical Engineering and Computer Science, The University of Newcastle, Callaghan, NSW 2308, Australia. Reza.Moheimani@newcastle.edu.au

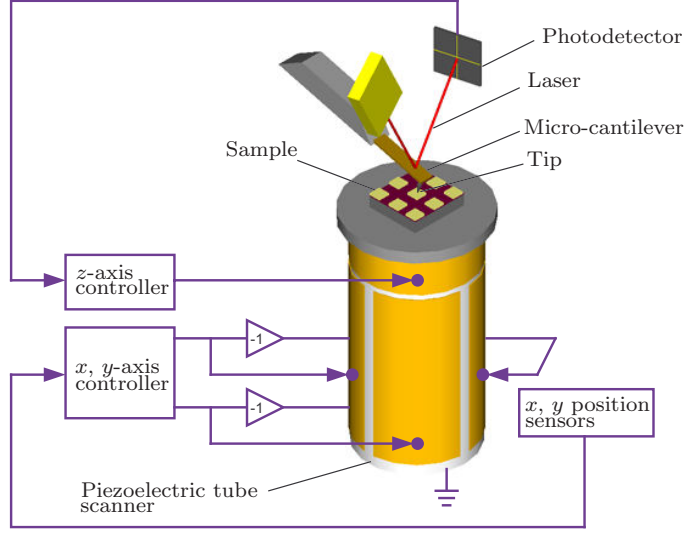

Fig. 1. Basic AFM schematic with feedback controllers.

piezoelectric tube actuator with quartered external electrodes for $3 \mathrm{D}$ positioning was first proposed in [3]. Today, the majority of commercially available AFMs use a piezoelectric tube scanner for $\mathrm{x}-\mathrm{y}-\mathrm{z}$ positioning, although flexure-based nanopositioners [4], [5], [6] are emerging as a viable, albeit more expensive alternative. Accuracy and speed of operation of an AFM are adversely affected by a number of properties inherent to piezoelectric materials.

Piezoelectric actuators exhibit hysteretic behavior. When a triangular voltage signal is applied to a piezoelectric actuator, the resulting displacement can deviate from linear by as much as $15 \%$ between the forward and backward movements. Earlier AFMs compensate for hysteresis effect by scanning always in the same direction [2]. In this openloop approach the input triangular signal is perturbed to achieve an acceptable trajectory, thus minimizing the effect of this particular form of nonlinearity. Modeling a piezoelectric actuator as a linear dynamic system cascaded with a static nonlinearity and then compensating for the nonlinearity through inversion is another approach that has been researched [7], [8], [9]. However, the authors are not aware if it has been incorporated into an existing AFM.

Another undesirable property of piezoelectric materials is creep. When the applied voltage to a piezoelectric actuator undergoes a sudden change, the piezoelectric material will creep. This can result in significant loss in precision when positioning is required over extended periods of time [10]. In particular during slow operation of atomic force microscopes, creep can result in significant distortions in the generated image [11]. Creep exacerbates the effect of hysteresis at the turning point of the scanning trajectory, and it has an adverse 
effect on the vertical positioning of the sample. While a number of methods have been proposed to deal with this phenomenon [12], [13], [14], [15], the most widely used approach in earlier AFMs has been to allow sufficient time for the effect of creep to disappear.

Yet another troublesome feature of AFMs, particularly in applications that involve nanoscale manipulation of materials, is the thermal drift of their mechanical setup. This arises from the thermal expansion and contraction of their mechanical components. In a typical AFM, operated in ambient temperature, a one degree change in temperature has been shown to cause as much as $50 \mathrm{~nm}$ drift [16]. This effect can be suppressed in low temperature experiments, e.g. if the AFM is operated in Ultra High Vacuum. However, in applications which involve interrogation or manipulation of the matter in ambient temperature it may amount to a substantial hurdle [16].

As indicated above, feedback has always been an integral part of every atomic force microscope for vertical positioning. A feedback controller requires a measurement signal to operate effectively. In an AFM, this measurement is conveniently made available by the photodetector, enabling accurate vertical positioning of the scanner. The utilization of feedback to improve lateral positioning of the scanner, however, requires displacement sensors to be incorporated into the device. These sensors were not included in scanners of earlier AFMs. However, they are progressively being built into a new generation of commercially available atomic force microscopes. The ability to use feedback for lateral positioning brings about a number of exciting possibilities some of which have already materialized in some state of the art AFMs. For example, high-gain feedback controllers have been used to reduce the effects of hysteresis, creep and thermal drift with significant success. Apart from the above complicating factors, there are two other issues that hamper the operation of an AFM: i) the highly resonant nature of the scanner, and ii) the cross coupling between the three axes of a piezoelectric tube.

To force the free end of the tube to move in a raster pattern, a triangular signal is applied to the $x$-axis (fast axis) electrodes and a slowly increasing ramp signal is applied to the $y$-axis (slow axis) electrodes. A triangular waveform contains all odd harmonics of the fundamental frequency. The amplitudes of these harmonic signals attenuate as $1 / n^{2}$, with $n$ being the harmonic number [17]. If a fast triangular waveform is applied to the scanner, it will inevitably excite the resonance. Consequently, instead of following a perfect triangle, the free end of the tube traces a distorted triangular waveform along the $x$-axis. This can significantly distort the image. To avoid this complication, the scanning speed of AFMs is often limited to about $1 \%$ of the scanner's first resonance frequency. A widely used approach to deal with this issue, during fast scans, is to shape the tracking signal such that it does not excite the tube's resonance. A downside of this approach is that the tube is still mechanically very lightly damped, and thus susceptible to external disturbances and noise.

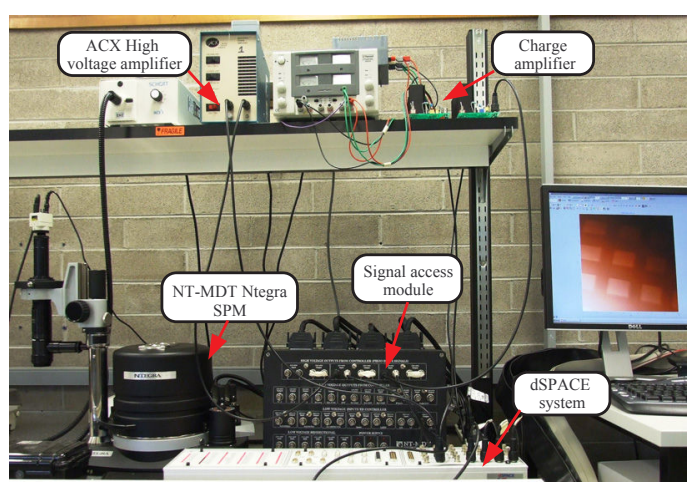

Fig. 2. SPM system and experimental setup used in this work

The existence of cross-coupling between the three axes of an AFM scanner can substantially distort the image. It can also be a major problem in applications that involve manipulation of matter at the nanoscale. Fig. 5 illustrates the lateral frequency responses of the AFM scanner used in our experiments. Significant cross-coupling can be observed between lateral axes of the scanner. For example, at low frequencies there is approximately $32 \mathrm{~dB}$ cross-coupling between $x$ and $y$ axes of the scanner. This means that a slow $8 \mu \mathrm{m}$ triangular motion of $x$ axis will translate into a $0.2 \mu \mathrm{m}$ triangular motion of the $y$ axis, generating substantial distortion in the resulting image. Also significant crosscoupling exists at, and close to the resonance frequency of the tube.

The remainder of the paper is arranged as follows. Section II provides descriptions of the AFM and other experimental setup used in this work. Modeling and identification of the system transfer functions are presented in Section III. Control schemes for the AFM scanner are devised in Section IV. In Section V experimental results are presented to illustrate the drastic improvement in accuracy and imaging speed that can be achieved with the proposed control schemes. Finally, Section VI concludes the paper.

\section{EXPERIMENTAL SETUP}

Our experimental setup consisted of a NT-MDT Ntegra scanning probe microscope (SPM) shown in Fig. 2 that was configured to operate as an AFM. The AFM was retrofitted with a home made charge source [18] on the fast axis. Charge drive significantly minimized the effect of hysteresis on this axis of the scanner. The slow axis was driven by an ACX High Voltage Amplifier. A dSPACE-1103 rapid prototyping system was used to implement the feedback controllers in real time. The amplifiers and the AFM were interfaced with the dSPACE system using a signal access module that allowed direct access to the scanner electrodes. This setup enabled us to directly control the lateral movements of the scanner. However, the vertical positioning was achieved using the AFM control software. In other words we replaced the rastering mechanism of the AFM with our own system.

The scanner is NT-MDT Z50309cl that performs 3D positioning in an NT-MDT Ntegra SPM. It is a piezoelectric 
tube scanner with quartered internal and external electrodes. Capacitive displacement sensors are incorporated into the scanner apparatus that allow for direct measurements of the tube displacement in $x, y$ and $z$ directions. A dSPACE rapid prototyping system equipped with ControlDesk software was interfaced to the AFM through an expansion box that allowed direct access to capacitive sensor measurements and control signals that were to be applied to the piezoelectric actuator. The recorded data were processed in Matlab to generate AFM images.

\section{SYSTEM IDENTIFICATION}

In order to simplify the control design, the AFM scanner was treated as two single-input single-output (SISO) systems. The inputs being the voltage signals applied to the charge amplifier, $u_{x}$, and to the voltage amplifier, $u_{y}$. The outputs of the systems are the tube displacement measurements in $x$ direction, $c_{x}$, and in $y$ directions, $c_{y}$. Accurate models of the systems were obtained through an experimental approach to modeling (system identification). The following frequency response functions (FRFs) were obtained nonparametrically using a dual-channel HP35670A spectrum analyzer:

$$
G_{c_{x} u_{x}}(i \omega)=\frac{c_{x}(i \omega)}{u_{x}(i \omega)}
$$

and

$$
G_{c_{y} u_{y}}(i \omega)=\frac{c_{y}(i \omega)}{u_{y}(i \omega)} .
$$

A band-limited random noise signal (1 to $1600 \mathrm{~Hz}$ ) was generated using the spectrum analyzer and applied to the amplifiers as the input. The corresponding outputs from the capacitive displacement sensors were also recorded using the spectrum analyzer. These input-output data were processed to generate the FRF (1) and (2) in a non-parametric form as illustrated in Fig. 3. Two second order models were fitted to the FRFs data using the frequency domain subspace-based system identification approach as described in [19] and [20]. The following transfer functions were found to be a good fit as illustrated in Fig. 3,

$$
G_{c_{x} u_{x}}(s)=\frac{0.05311 s^{2}-1230 s+1.362 \times 10^{7}}{s^{2}+40.38 s+1.354 \times 10^{7}}
$$

and

$$
G_{c y u_{y}}(s)=\frac{0.0849 s^{2}-1416 s+1.288 \times 10^{7}}{s^{2}+61.74 s+1.303 \times 10^{7}} .
$$

\section{Controller Design}

To address the issues discussed in Section I, we designed feedback controllers to augment the damping of the scanner's transfer functions to achieve improved lateral positioning. Structure of the $x$ axis feedback controller is illustrated in Fig. 4. A similar controller was designed for the $y$ axis. In our experiments, the fast axis was driven by a DC-accurate charge amplifier. Piezoelectric actuators are often driven by voltage amplifiers. Voltage-driven piezoelectric materials are known to display hysteretic behavior. An interesting, and
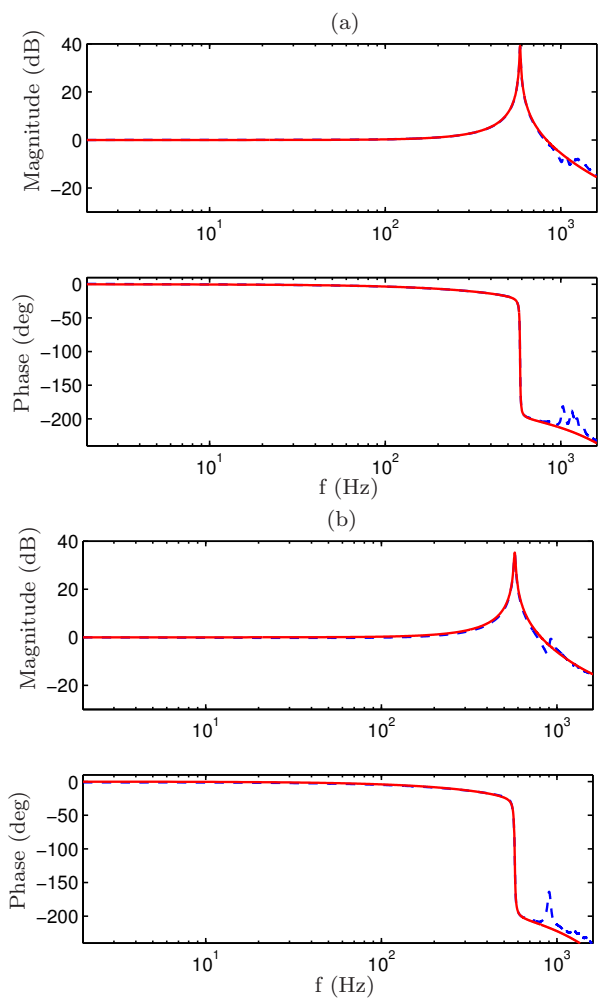

Fig. 3. Experimental (--) and identified model (-) frequency response of (a) $G_{c_{x} u_{x}}(i \omega)$ and (b) $G_{c_{y} u_{y}}(i \omega)$.

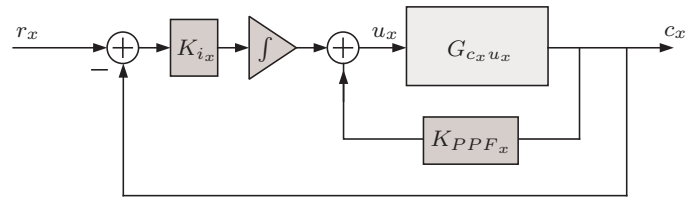

Fig. 4. Structure of the $x$ axis feedback controller. The inner feedback loop is a positive position feedback (PPF) controller designed to damp the highly resonant mode of the tube. Integral action is also incorporated to achieve satisfactory tracking.

unique property of piezoelectric materials is that when driven by a charge source, they display minimal hysteresis. This property has been known since 1980s [21], [22]. However, until very recently, it has been rarely used due to the difficulties associated with driving highly capacitive loads with commercially available charge or current amplifiers. Recently, we proposed [23], [24], [25], [18] a new construction for charge and current sources capable of regulating the DC profile of the actuator.

The overall control structure, illustrated in Fig. 4, consists of two feedback loops. The inner loop contains a Positive Position Feedback (PPF) controller that works to increase mechanical damping of the tube. PPF controllers were initially designed to suppress mechanical vibrations of highly resonant aerospace structures [26]. They have been successfully implemented on a range of lightly damped structures [27], [28], [29]. Their effectiveness in improving accuracy and bandwidth of nanopositioning systems was 

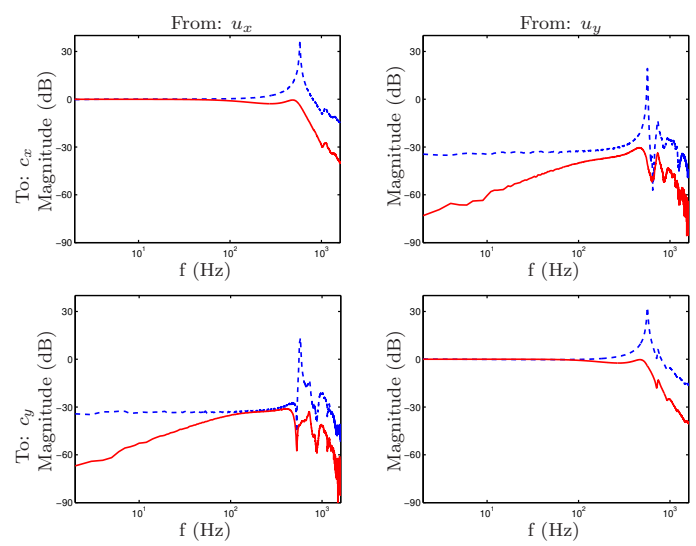

Fig. 5. Open-loop (blue) and closed-loop (red) Frequency responses of the scanner. The resonant behavior of the scanner is improved by over $30 \mathrm{~dB}$ due to control action. The proposed feedback control strategy results in significant improvement in cross-coupling between the fast axis and the slow axis of the scanner.

recently investigated in reference [30]. PPF controllers have a number of important features. In particular, they have a simple structure, are easy to implement and their transfer functions roll off at a rate of $40 \mathrm{~dB} /$ decade at higher frequencies. The latter is important in terms of the overall effect of sensor noise on the scanner's positioning accuracy. The details of the procedure that was followed to design these PPF controllers is documented in reference [30]. The obtained PPF controllers can be described as

$$
K_{P P F_{x}}(s)=\frac{9.242 \times 10^{6}}{s^{2}+6032 s+2.785 \times 10^{7}}
$$

and

$$
K_{P P F_{y}}(s)=\frac{9.499 \times 10^{6}}{s^{2}+6062 s+2.753 \times 10^{7}} .
$$

The control system we designed also includes integral action as illustrated in Fig. 4. Inclusion of an integrator amounts to applying a high gain at low frequencies that reduces the effects of thermal drift, piezoelectric creep and hysteresis to a minimum. An important benefit of the proposed combined feedback structure is the significant reduction that can be achieved in cross-coupling between various axes of the scanner. The gain of the integrators were tuned to provide high closed-loop bandwidth but with reasonable gain and phase margin.

\section{Results}

The performance of the control schemes were first evaluated by measuring the closed-loop responses of the AFM scanner using the spectrum analyzer. In Fig. 5, the closedloop frequency responses are plotted along with the openloop frequency responses. By examining the frequency responses we can see that the closed-loop system bandwidth of both axes is about $300 \mathrm{~Hz}$. Also, a damping of more than $30 \mathrm{~dB}$ at each resonant mode is apparent from the frequency responses.

To appreciate the improvement achieved in lateral positioning of the scanner, we performed a simple experiment.
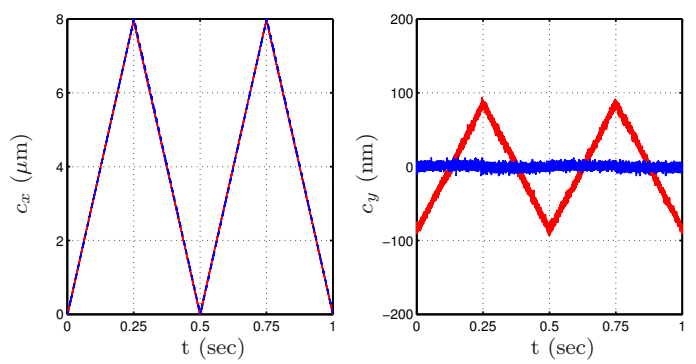

(a)
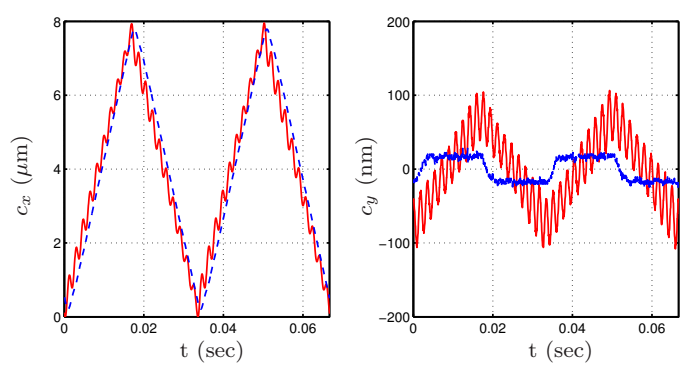

(b)

Fig. 6. Closed-loop (blue) and open-loop (red) tracking performance (left) and cross-coupling properties (right) of the scanner for (a) $2 \mathrm{~Hz}$ scan, and (b) $30 \mathrm{~Hz}$ scan.

A $2 \mathrm{~Hz}$ triangular signal was applied to the fast axis of the piezoelectric tube to achieve an $8 \mu \mathrm{m}$ scan. The subsequent displacements of the slow axis and vertical axis of the tube were measured using the built-in capacitive displacement sensor. A similar experiment was then carried out with the feedback controllers implemented on the tube. The results are plotted in Fig. 6(a). A significant improvement can be observed by comparing the open-loop and closed-loop motions of the tube. A similar set of experiments were performed at $30 \mathrm{~Hz}$ to simulate a fast scan. The results are plotted in Fig. 6(b). During a fast scan we can observe significant distortions due to the excitation of the tube's resonance. By applying the feedback controller, we managed to significantly reduce the distortions and achieved a remarkable improvement in tracking performance. In particular, for the $30 \mathrm{~Hz}$ scan, the fast axis RMS tracking error was reduced from $280 \mathrm{~nm}$ to $46 \mathrm{~nm}$, and the cross-coupling to the slow axis was reduced from $50 \mathrm{~nm}$ to $16 \mathrm{~nm}$ due to control action. This improvement directly translates into an image with less distortion. A significant component of the closed-loop error is due to the phase shift between the desired and the achieved trajectories. This phase shift has a minimal effect on the image quality and can be handled using a feedforward controller.

Having improved the lateral positioning of the scanner, we then moved on to investigate the overall improvement in imaging capability of our modified AFM. During the imaging, the atomic force microscope was operated in constant force mode using a micro-cantilever with spring constant of $0.2 \mathrm{~N} / \mathrm{m}$. The sample was a $20 \mathrm{~nm}$ feature-height NTMDT TGQ1 calibration grating with a $3 \mu \mathrm{m}$ pitch. The 

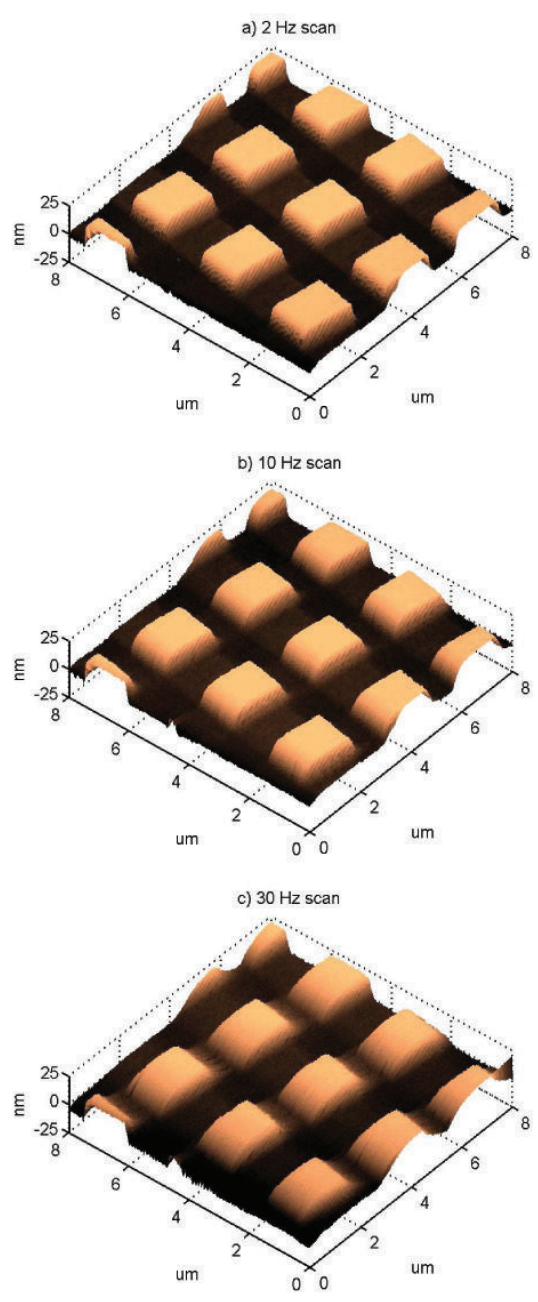
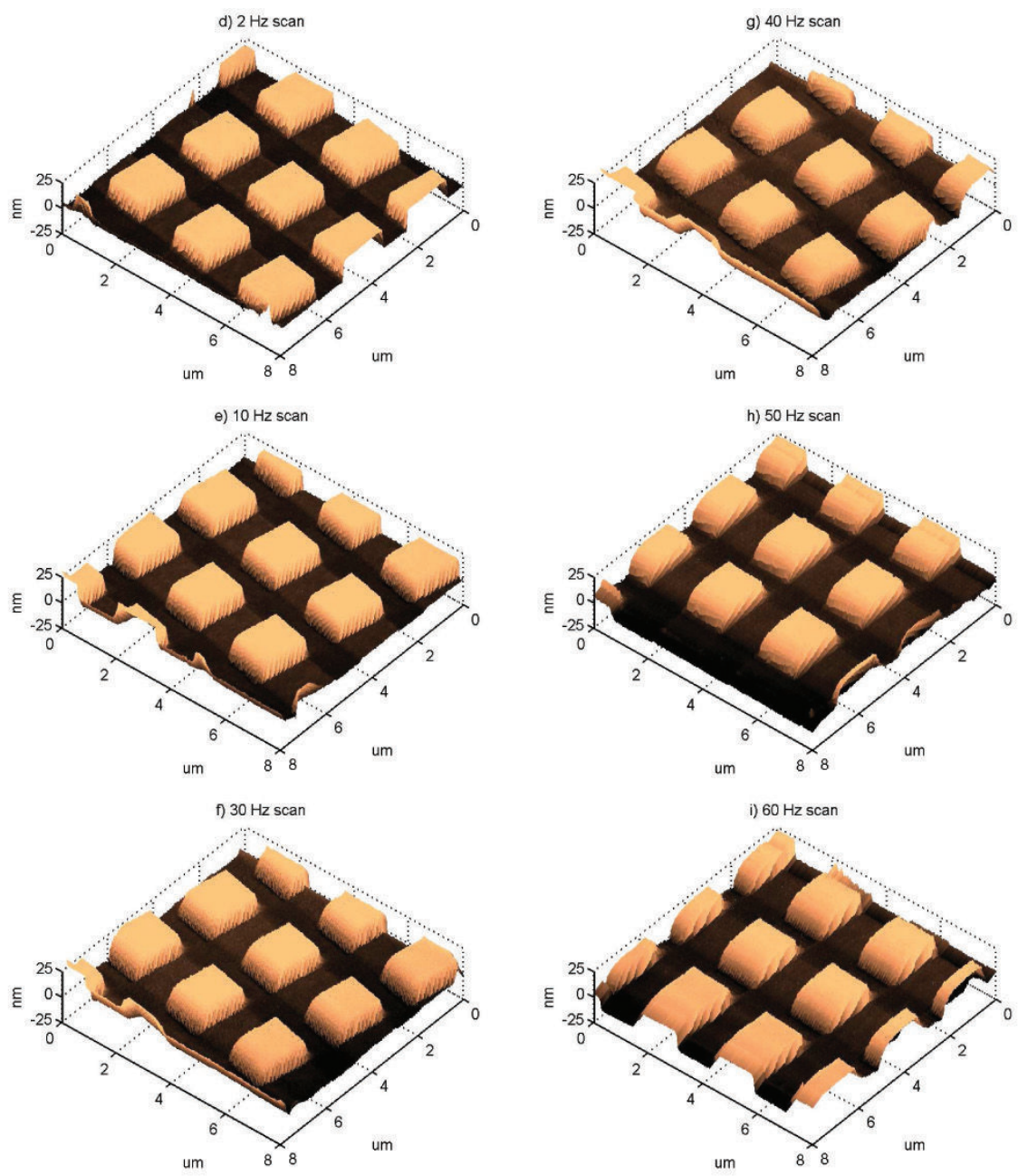

Fig. 7. First two columns: AFM images of NT-MDT TGQ1 grating scanned in contact mode constant force at 2, 10 and $30 \mathrm{~Hz}$. Images displayed in (a), (b) and (c) were developed using the AFM controller. Images displayed in (d), (e) and (f) were generated using the PPF controller. A significant improvement in image quality can be observed. Third column: We were able to generate images at scan frequencies beyond the AFM limit of $30 \mathrm{~Hz}$. 40 , 50 and $60 \mathrm{~Hz}$ scans are illustrated in $(\mathrm{g})$, (h) and (i) respectively.

AFM controller was first used to develop $8 \mu \mathrm{m} \times 8 \mu \mathrm{m}$ images of the sample at $2 \mathrm{~Hz}, 10 \mathrm{~Hz}$ and $30 \mathrm{~Hz}$ scan frequencies with a resolution of $256 \times 256$ pixels. Faster scans beyond $30 \mathrm{~Hz}$ with the AFM software, at this resolution, were not possible. In each case, a significant amount of time was devoted to tune the AFM so that the best possible image could be generated. We then imaged the sample at identical frequencies, but this time with our own feedback controller implemented on the scanner. These images are plotted in Fig. 7 for comparison, and illustrate a drastic improvement in image quality and sharpness. Furthermore, our modifications enabled us to scan beyond the $30 \mathrm{~Hz}$ speed set by the AFM controller. In particular, we developed scans of the sample at $40 \mathrm{~Hz}, 50 \mathrm{~Hz}$ and $60 \mathrm{~Hz}$ with the same resolution. These results are also plotted in Fig. 7.

\section{COnclusions And Future Work}

In conclusion, we demonstrated that by augmenting the damping of an AFM scanner, and minimizing the crosscoupling between its fast and slow axes, and also by using charge drive on its fast axis, the quality of the developed image could be drastically improved. This is a direct result of the improvement in lateral positioning of the AFM scanner. We were also able to scan at frequencies beyond the limit set by the AFM software. Future research will involve minimizing cross coupling to the vertical axis of the scanner to achieve higher resolution images at faster scans.

\section{REFERENCES}

[1] G. Binnig, C. F. Quate, and C. Gerber, "Atomic force microscope," Physical Review Letters, vol. 56, no. 9, pp. 930-933, March 1986.

[2] E. Meyer, H. J. Hug, and R. Bennewitz, Scanning Probe Microscopy. Heidelberg, Germany: Springer, 2004.

[3] G. Binnig and D. P. E. Smith, "Single-tube three-dimensional scanner for scanning tunneling microscopy," Review of Scientific Instruments, vol. 57, no. 8, pp. 1688-1689, August 1986.

[4] S. Salapaka, A. Sebastian, J. P. Cleveland, and M. V. Salapaka, "High bandwidth nano-positioner: A robust control approach," Review of Scientific Instruments, vol. 73, no. 9, pp. 3232-3241, September 2002.

[5] A. Sebastian and S. M. Salapaka, "Design methodologies for robust nano-positioning," IEEE Trans. Contr. Syst. Technol., vol. 13, no. 6, pp. 868-876, November 2005. 
[6] S. Aphale, B. Bhikkaji, and S. O. R. Moheimani, "Minimizing scanning errors in piezoelectric stack-actuated nanopositioning platforms," IEEE Trans. Nanotechnol., vol. 7, no. 9, pp. 79-90, January 2008.

[7] P. Ge and M. Jouaneh, "Modeling hysteresis in piezoceramic actuators," Precision Engineering, vol. 17, pp. 211-221, 1995.

[8] R. B. Mrad and H. Hu, "A model for voltage to displacement dynamics in piezoceramic actuators subject to dynamic voltage excitations," IEEE/ASME Trans. Mechatronics, vol. 7, no. 4, pp. 479-489, December 2002.

[9] S. H. Lee and T. J. Royston, "Modeling piezoceramic transducer hysteresis in the structural vibration control problem," J. Acoust. Soc. Am., vol. 108, no. 6, pp. 2843-2855, December 2000.

[10] T. Fett and G. Thun, "Determination of room-temperature tensile creep of pzt," Journal of Materials Science Letters, vol. 17, no. 22, pp. 19291931, 1998.

[11] R. S. Robinson, "Interactive computer correction of piezoelectric creep in scanning tunneling microscopy images," Journal of ComputerAssisted Microscopy, vol. 2, no. 1, pp. 53-58, 1996.

[12] H. Jung, J. Y. Shim, and D. Gweon, "New open-loop actuating method of piezoelectric actuators for removing hysteresis and creep," Review of Sicentific Instruments, vol. 71, no. 9, pp. 3436-3440, September 2000.

[13] S. Salapaka, A. Sebastian, J. P. Cleveland, and M. V. Salappaka, "Design, identification and control of a fast nanopositioning device," in Proc. American Control Conference, May 2002, pp. 1966-1971.

[14] D. Croft, G. Shed, and S. Devasia, "Creep, hysteresis, and vibration compensation for piezoactuators: Atomic force microscopy application," ASME Journal of Dynamic Systems and Control, vol. 123, no. 1, pp. 35-43, March 2001

[15] S. Devasia, E. Eleftheriou, and S. O. R. Moheimani, "A survey of control issues in nanopositioning," IEEE Trans. Contr. Syst. Technol., vol. 15, no. 5, pp. 802-823, September 2007.

[16] B. Mokaberi and A. G. Requicha, "Drift compensation for automatic nanomanipulation with scanning probe microscopes," IEEE Trans. Autom. Sci. Eng., vol. 3, p. 3, July 2006.

[17] B. P. Lathi, Linear Systems and Signals, 2nd ed. Oxford University Press, 2004

[18] A. J. Fleming and S. O. R. Moheimani, "Sensorless vibration suppression and scan compensation for piezoelectric tube nanopositioners," IEEE Trans. Contr. Syst. Technol., vol. 14, no. 1, pp. 33-44, 2006.
[19] T. McKelvey, H. Ackay, and L. Ljung, "Subspace-based identification of infinite-dimensional multi-variable systems from frequencyresponse data," vol. AC41, pp. 960-979, 1996.

[20] T. McKelvey, A. J. Fleming, and S. O. R. Moheimani, "Subspacebased system identification for an acoustic enclosure," Transactions of the ASME Journal of Vibration and Acoustics, vol. 124, no. 3, pp. 414-419, July 2002.

[21] R. Comstock, "Charge control of piezoelectric actuators to reduce hysteresis effect," U.S. Patent 4.263,527, 1981.

[22] C. Newcomb and I. Flinn, "Improving the linearity of piezoelectric actuators using charge feedback," Electronics Letters, vol. 18, no. 11, pp. 442-444, May 1982.

[23] A. J. Fleming and S. O. R. Moheimani, "Precision current and charge amplifiers for driving highly capacitive piezoelectric loads,' Electronics Letters, vol. 39, no. 3, pp. 282-284, February 2003.

[24] _ - "Improved current and charge amplifiers for driving piezoelectric loads, and issues in signal processing design for synthesis of shunt damping circuits," Journal of Intelligent Material Systems and Structures, vol. 15, no. 2, pp. 77-92, February 2004.

[25] — "A grounded load charge amplifier for reducing hysteresis in piezoelectric tube scanners," Review of Scientific Instruments, vol. 76, no. 7, 2005

[26] J. L. Fanson and T. K. Caughey, "Positive position feedback-control for large space structures," AIAA Journal, vol. 28, no. 4, pp. 717-724, April 1990

[27] K. H. Rew, J. H. Han, and I. Lee, "Multi-modal vibration control using adaptive positive position feedback," Joural of Intelligent Material Systems and Structures, vol. 13, no. 1, pp. 13-22, January 2002.

[28] G. Song, S. P. Schmidt, and B. N. Agrawal, "Experimental robustness study of positive position feedback control for active vibration suppression," Journal of Guidance, Control and Dynamics, vol. 25, no. 1 , pp. 179-182, 2002.

[29] S. O. R. Moheimani, B. J. G. Vautier, and B. Bhikkaji, "Experimental implementation of extended multivariable PPF control on an active structure," IEEE Trans. Contr. Syst. Technol., vol. 14, no. 3, pp. $443-$ 445, May 2006

[30] B. Bhikkaji, M. Ratnam, A. J. Fleming, and S. O. R. Moheimani, "High-performance control of piezoelectric tube scanners," IEEE Trans. Contr. Syst. Technol., vol. 5, no. 5, pp. 853-866, September 2007. 